\title{
Misleading Numbers: Is the Risk of Acute Kidney Injury with COVID-19 Truly This Low?
}

\author{
Samuel A. Silver ${ }^{\mathrm{a}}$ Edward G. Clark ${ }^{\mathrm{b}}$ Swapnil Hiremath ${ }^{\mathrm{b}}$ \\ aDivision of Nephrology, Department of Medicine, Queen's University, Kingston, ON, Canada; \\ ${ }^{b}$ Division of Nephrology, Department of Medicine, University of Ottawa, Ottawa, ON, Canada
}

\section{Dear Editor,}

The case series from Wang et al. [1] of 116 patients from Remnin Hospital with coronavirus disease 2019 (COVID-19) reports that none developed acute kidney injury (AKI). This finding and several other aspects of this study cause us concern. In particular, $0 \%$ of patients with AKI is strikingly inconsistent with the reported literature so far. In addition, two other case series published as preprints from the same institution reported that 23$32 \%$ of deaths were accompanied by AKI $[2,3]$. We speculate that this discrepancy may have occurred for several reasons.

First, as it is unclear how the authors assembled this cohort, it is not possible to determine which fraction of patients admitted to the institution was included. Was it a specialized COVID-19 ward for a selected patient population? Without more details, it is possible that this case series consists of a highly selected group of patients, and hence is not representative of the general patient population. This is suggested by the small number of patients with any preexisting comorbidities. Although 5 patients had end-stage kidney disease at baseline, none of the others had CKD despite a definition of CKD which was quite broad. Another potential source of selection bias is that it is unclear where patients were captured with respect to the trajectory of their COVID-19 infections. Were they all new

\section{KARGER}

(c) 2020 S. Karger AG, Basel admissions? Were some patients already hospitalized at the time of their COVID-19 diagnosis? Were some already recovering? The patients most at risk for AKI would be those captured during the acute phase of their illness. Without having more details about how patients were identified for inclusion, the results are difficult to interpret.

Second, no data are provided regarding the number of patients who had a baseline serum $\mathrm{Cr}$ available. These definitions result in bidirectional misclassification of AKI incidence, and so clear numbers are needed $[4,5]$.

Third, outcome ascertainment for AKI is unclear. It is unclear how often serum $\mathrm{Cr}$ and urine output were measured to ascertain AKI. To some extent, the availability of renal replacement therapy could have affected ascertainment if patients died with (or recovered from) AKI before having had follow-up kidney function testing. Table 2 [1] from the study suggests that testing was done weekly, which may not have been sufficiently frequent in certain instances. Additionally, it is unclear how to interpret what is meant by serum Cr values and kidney deterioration for the 5 patients with end-stage kidney disease who already were on dialysis.

Last, given that AKI affects between 10 and 25\% of hospitalized patients, we would expect patients with $\mathrm{CO}$ VID-19 to at least fall somewhere within this range or above it if specifically assessing a critically ill population.

Prof. Swapnil Hiremath 
For the reasons stated above, we believe that the results reported by Wang et al. [1] should be taken with caution. We suggest that clinicians should continue to closely monitor for AKI in patients with COVID-19 as they would in other hospitalized patients and while we await further evidence of any potentially unique AKI risks in this population.

\section{Acknowledgement}

E.G.C. and S.H. would like to acknowledge research salary support from the Department of Medicine, University of Ottawa.

\section{Disclosure Statement}

The authors have no conflicts of interest to declare.

\section{Funding Sources}

The authors did not receive any funding.

\section{Author Contributions}

The letter was drafted by all three authors.

\section{References}

1 Wang L, Li X, Chen H, Yan S, Li D, Li Y, et al. Coronavirus disease 19 infection does not result in acute kidney injury: an analysis of 116 hospitalized patients from Wuhan, China. Am J Nephrol. 2020;51(5):343-8.

2 Zhang B, Zhou X, Qiu Y, Feng F, Feng J, Jia Y, et al. Clinical characteristics of 82 death cases with COVID-19[Internet]. Infectious Diseases (except HIV/AIDS). medRxiv; 2020. Available from: https://www.medrxiv.org/content /10.1101/2020.02.26.20028191v1.
3 Shi Q, Zhao K, Yu J, Feng J, Zhao K, Zhang X, et al. Clinical characteristics of 101 non-surviving hospitalized patients with COVID-19: a single center, retrospective study[Internet]. Infectious Diseases (except HIV/AIDS). medRxiv; 2020. Available from: https://www. medrxiv.org/content/10.1101/2020.03.04.20 031039v2
4 Siew ED, Matheny ME. Choice of reference serum creatinine in defining acute kidney injury. Nephron. 2015 Sep 2;131(2):107-12.

5 Siew ED, Ikizler TA, Matheny ME, Shi Y, Schildcrout JS, Danciu I, et al. Estimating baseline kidney function in hospitalized patients with impaired kidney function. Clin J Am Soc Nephrol. 2012 May;7(5):712-9. 